\title{
Photometric and period investigation of the late F-type overcontact binary II UMa
}

\author{
X. Zhou ${ }^{1,2,3}$, S.-B. Qian ${ }^{1,2,3}$, J. Zhang ${ }^{1,2}$, B. Zhang ${ }^{1,2,3}$, J. Kreiner ${ }^{4}$
}

\begin{abstract}
II UMa is a late F-type (F5) contact binary with a close-in tertiary and a distant visual companion. According to the four-color $\left(B V R_{c} I_{c}\right)$ light curves' solutions of II UMa, it is a high fill-out $(\mathrm{f}=86.6 \%)$ and low mass ratio $(q=$ 0.172 ) contact binary system, which indicate that it is at the late evolutionary stage of late-type tidal-locked binary stars. The mass of the primary star and secondary one are calculated to be $M_{1}=1.99 M_{\odot}, M_{2}=0.34 M_{\odot}$. The primary star has evolved from ZAMS, but it is still before TAMS, and the secondary star is even more evolved. Considering the mass ratio $\left(M_{3} / M_{1}=0.67\right)$ obtained by spectroscopic observations, the mass of the close-in tertiary is estimated to be $M_{3}=1.34 M_{\odot}$. The period variations of the binary system is investigated for the first time. According to the observed-calculated $(O-C)$ curve analysis, a continuous period increase at a rate of $d P / d t=4.88 \times 10^{-7}$ day $\cdot$ year $^{-1}$ is determined. It may be just a part of a cyclic period change, or the combinational period change of a parabolic variation and a cyclic one. More times of minimum light are needed to confirm this. The presence of the tertiary component may play an important role in the formation and evolution of this binary system by drawing angular momentum from the central system during the pre-contact stage.
\end{abstract}

Subject headings: binaries : close - binaries : eclipsing - stars: evolution - stars: individuals (II UMa)

\footnotetext{
${ }^{1}$ Yunnan Observatories, Chinese Academy of Sciences (CAS), P. O. Box 110, 650216 Kunming, China; zhouxiaophy@ynao.ac.cn

${ }^{2}$ Key Laboratory of the Structure and Evolution of Celestial Objects, Chinese Academy of Sciences, P. O. Box 110, 650216 Kunming, China

${ }^{3}$ University of Chinese Academy of Sciences, Yuquan Road 19\#, Sijingshang Block, 100049 Beijing, China

${ }^{4}$ Mt. Suhora Astronomical Observatory, Pedagogical University of Cracow, Poland
} 


\section{Introduction}

W UMa type binaries are cool short-period (usually less than 1 day) binary systems with both components filling their critical Roche lobes and sharing a common convective envelope during their main sequence (MS) evolutionary stage. The more massive primary component is a main sequence star whereas the secondary is oversized compared to its expected MS radius. The formation and evolution of W UMa type binary systems are still unsolved problems in astrophysics. The most popular evolutionary scenario is that they are formed from initially detached systems via angular momentum loss (AML) by means of magnetic stellar wind (Vilhu 1982; Eggen \& Iben 1989). Model calculations suggest that these binary stars will ultimately coalesce into single stars which may be progenitors of the poorly understood blue stragglers and FK Com-type stars (Stẹpień 2006, 2011).

II UMa (BD+55 1540, HIP 61237, $\left.V=8^{m} .48\right)$ is a component of the visual binary ADS 8954 , with the separation of $0^{\prime \prime} .87$ and difference in brightness of 1.64 mag. The photometric variability of the star was discovered by the Hipparcos satellite (ESA, 1997). Radial velocity

curves of both components in II UMa were obtained by Rucinski et al. (2002) and gave the results: $q=0.172 \pm 0.004, V_{\gamma}=-8.02 \pm 1.10 \mathrm{~km} \mathrm{~s}^{-1},\left(M_{1}+M_{2}\right) \sin ^{3} i=2.180 \pm 0.080 M_{\odot}$. They pointed out that II UMa was a A-subtype W UMa binary system with a spectral type of F5III. Later, detailed investigation by D'Angelo et al. (2006) determined that it was a triple system with a solar-type close-in tertiary component. The temperature of the tertiary component is about $6100 \mathrm{~K}$ and the mass ratio is $M_{3} / M_{1}=0.67$. The first photometric solutions of II UMa were published by Oh et al. (2007). Then, Yllmaz et al. (2015) also obtained the photometric parameters of this binary system. In the present paper, four-color light curves (LCs) of II UMa are analyzed and its formation and evolutionary scenario are discussed. The period variations of the binary system is investigated for the first time, which may reveal the dynamics interaction between the two components.

\section{Observations}

The four-color ( $B V R_{c} I_{c}$ ) light curves of II UMa were carried out in five continuous nights on February 1, February 2, February 3, February 4 and February 5, 2012 with an Andor DV436 2K CCD camera attached to the $60 \mathrm{~cm}$ reflecting telescope at Yunnan Observatories (YNOs). The coordinates of the variable star, the comparison star and the check star were listed in Table 1. The integration time were $60 \mathrm{~s}$ for $B$ band, 30s for $V$ band, 15 s for $R_{c}$ band, and 10s for $I_{c}$ band, respectively. The light curves of those observations were displayed in Fig. 1. During the observations, the broadband Johnson-Cousins $B V R_{c} I_{c}$ filters were used. PHOT (measured magnitudes for a list of stars) of the aperture photometry package 
in the IRAF 1 was used to reduce the observed images.

Table 1: Coordinates of II UMa, the comparison and the check stars.

\begin{tabular}{ccccc}
\hline \hline Targets & name & $\alpha_{2000}$ & $\delta_{2000}$ & $V_{\text {mag }}$ \\
\hline Variable & II UMa & $12^{h} 32^{m} 54^{s} .8$ & $+54^{\circ} 47^{\prime} 42^{\prime \prime} .9$ & 8.26 \\
The comparison & GSC 03841-00375 & $12^{h} 32^{m} 16^{s} .6$ & $+54^{\circ} 47^{\prime} 57^{\prime \prime} .5$ & 10.05 \\
The check & GSC 03841-00259 & $12^{h} 32^{m} 19^{s} .2$ & $+54^{\circ} 53^{\prime} 21^{\prime \prime} .7$ & 11.15 \\
\hline \hline
\end{tabular}

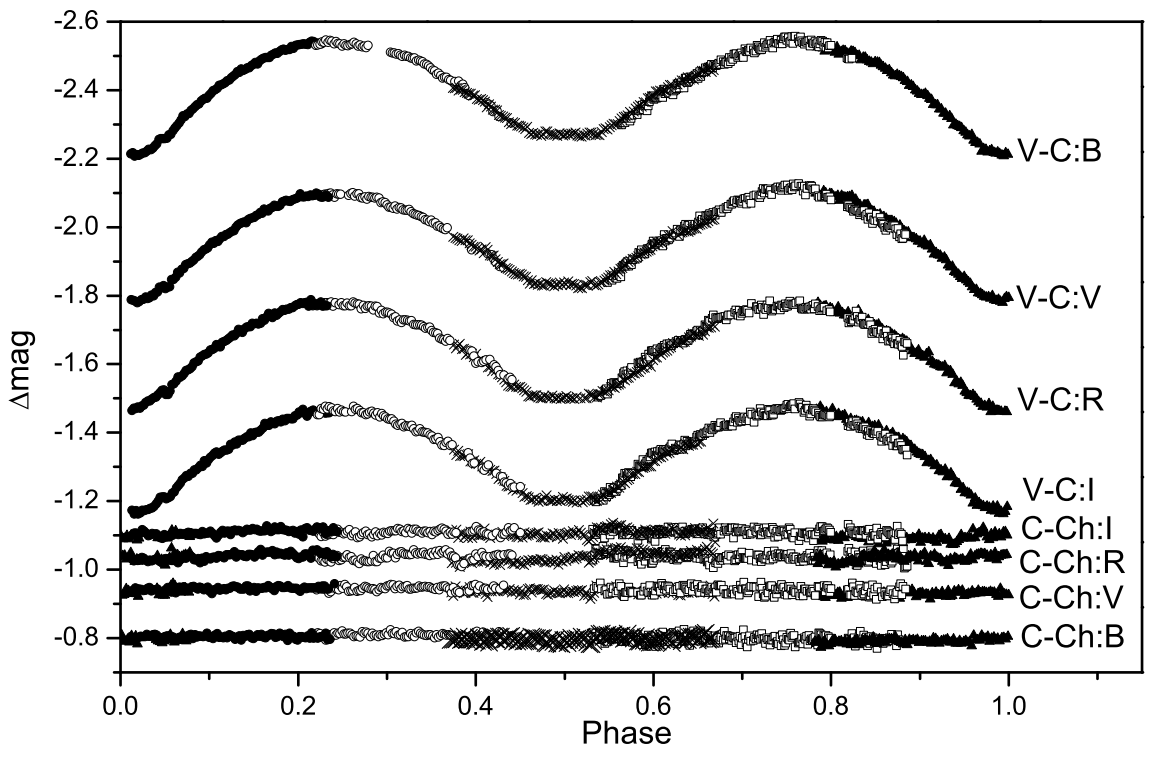

Fig. 1. - CCD photometric light curves in $B V R_{c}$ and $I_{c}$ bands. The magnitude difference between the comparison and the check stars are presented. The standard deviations of the comparison-check observations are $0.010 \mathrm{mag}$ for $B$ band, $0.010 \mathrm{mag}$ for $V$ band, $0.012 \mathrm{mag}$ for $R_{c}$ band and 0.011 mag for $I_{c}$ band. Solid circles, open circles, crosses, squares and triangles correspond to the data observed on February 1, February 2, February 3, February 4 and February 5, 2012, respectively.

Times of minimum light of II UMa were also observed and determined, which were listed in Table 2 ,

\footnotetext{
${ }^{1}$ The Image Reduction and Analysis Facility is hosted by the National Optical Astronomy Observatories in Tucson, Arizona at URL iraf.noao.edu.
} 
Table 2: New CCD times of light minimum for II UMa.

\begin{tabular}{cccccc}
\hline JD (Hel.) & Error (days) & Min. & Filter & Method & Telescopes \\
\hline 2455961.3376 & \pm 0.0005 & II & $B V R_{c} I_{c}$ & CCD & $60 \mathrm{~cm}$ \\
2455963.3963 & \pm 0.0005 & I & $B V R_{c} I_{c}$ & CCD & $60 \mathrm{~cm}$ \\
2456030.2416 & \pm 0.0002 & I & $N$ & CCD & $60 \mathrm{~cm}$ \\
2456265.4368 & \pm 0.0011 & I & $R_{c} I_{c} N$ & CCD & $60 \mathrm{~cm}$ \\
2456399.1215 & \pm 0.0008 & I & $V R_{c} I_{c} N$ & CCD & $60 \mathrm{~cm}$ \\
2456404.0720 & \pm 0.0007 & I & $V R_{c} I_{c} N$ & CCD & $60 \mathrm{~cm}$ \\
2456744.0597 & \pm 0.0007 & I & $V R_{c} I_{c}$ & CCD & $1 \mathrm{~m}$ \\
\hline
\end{tabular}

Notes. $60 \mathrm{~cm}$ and $1 \mathrm{~m}$ denote to the $60 \mathrm{~cm}$ and $1 \mathrm{~m}$ reflecting telescope in Yunnan Observatories.

\section{Orbital Period Investigation}

The study of orbital period change is a very important part for contact binary stars. However, the period change investigation of II UMa has been neglected since it was discovered. During the present work, all available times of minimum light are collected. Minimum times with the same epoch have been averaged, and only the mean values are listed in Table 3.

The minimum times of II UMa are listed in Table 3, Using the following linear ephemeris,

$$
\text { Min.I }(H J D)=2456030.2416+0^{d} .82522 \times E,
$$

the $O-C$ values are calculated and listed in the fourth column of Table 3 and plotted in the upper panel of Fig. 2. Based on the least-square method, the new ephemeris is

$$
\begin{aligned}
& \text { Min.I }=2456030.2428( \pm 0.0001)+0.82522813( \pm 0.00000006) \times E \\
& +0.552( \pm 0.007) \times 10^{-9} \times E^{2}
\end{aligned}
$$

With the quadratic term included in this ephemeris, a continuous period increase, at a rate of $d P / d t=4.88 \times 10^{-7} d a y \cdot y e a r^{-1}$ is determined. The residuals from Equation (2) are displayed in the lower panel of Fig. 2.

\section{Photometric Solutions}

II UMa is an EW-type contact binary. The W-D program of 2013 version (Wilson \& Devinnev 1971; Wilson 1979, 1990; Van Hamme \& Wilson 2007; Wilson 2008; Wilson et al. 2010; Wilson 2012) are used in modeling the light curves. The number of observational data 
Table 3: $(O-C)$ values of minimum light for II UMa.

\begin{tabular}{cclllll}
\hline \hline JD (Hel.) & Min & Epoch & $(O-C)$ & Error & Method & Reference \\
$(2400000+)$ & & & & & & \\
\hline 48371.7587 & II & -9280.5 & -0.0287 & 0.0014 & CCD & 1 \\
48372.1747 & I & -9280 & -0.0253 & 0.0016 & CCD & 1 \\
51221.6579 & I & -5827 & -0.0268 & 0.0044 & CCD & 2 \\
52649.7056 & II & -4096.5 & -0.0223 & 0.0007 & CCD & 3 \\
52723.5654 & I & -4007 & -0.0197 & 0.0004 & CCD & 4 \\
53064.3778 & I & -3594 & -0.0231 & 0.0002 & CCD & 5 \\
53081.7130 & I & -3573 & -0.0176 & 0.0020 & CCD & 6 \\
53761.2818 & II & -2749.5 & -0.0174 & & CCD & 7 \\
53809.1465 & II & -2691.5 & -0.0155 & & CCD & 7 \\
54528.3212 & I & -1820 & -0.0200 & & CCD & 8 \\
55231.8365 & II & -967.5 & -0.0048 & 0.0001 & CCD & 9 \\
55280.5232 & II & -908.5 & -0.0060 & 0.0003 & CCD & 10 \\
55961.3376 & II & -83.5 & 0.0018 & 0.0005 & CCD & 11 \\
55963.3963 & I & -81 & -0.0025 & 0.0005 & CCD & 11 \\
56030.2416 & I & 0 & 0 & 0.0002 & CCD & 11 \\
56057.4753 & I & 33 & 0.0014 & 0.0004 & CCD & 12 \\
56265.4368 & I & 285 & 0.0075 & 0.0011 & CCD & 11 \\
56319.4864 & II & 350.5 & 0.0052 & 0.0006 & CCD & 12 \\
56399.1215 & I & 447 & 0.0066 & 0.0008 & CCD & 11 \\
56404.0720 & I & 453 & 0.0057 & 0.0007 & CCD & 11 \\
56713.5344 & I & 828 & 0.0106 & 0.0007 & CCD & 12 \\
56725.4998 & II & 842.5 & 0.0103 & 0.0002 & CCD & 12 \\
56744.0597 & I & 865 & 0.0027 & 0.0007 & CCD & 11 \\
\hline & & & & & & \\
\hline
\end{tabular}

Reference: (1) private provision; (2) Rucinski et al. (2002); (3) Drozdz \& Ogloza (2005); (4) Porowski (2005); (5) Krajci (2005); (6) Nelson (2005); (7) K. Nagai (2007); (8) K. Nagai (2009); (9) Dvorak (2011); (10) Brat et al. (2011); (11) present work; (12) Zasche et al. (2014)

points used in W-D program are 566 in $B$ band, 599 in $V$ band, 613 in $R_{c}$ band, 633 in $I_{c}$ band, respectively. The phases are calculated with the following linear ephemeris:

$$
\text { Min.I }(H J D)=2456030.2416+0^{d} .82522 \times E .
$$

According to its spectral type of $\mathrm{F} 5$, the effective temperature of star 1 is assumed to be $T_{1}=6550 \mathrm{~K}$. The bolometric albedo $A_{1}=A_{2}=0.5$ (Ruciński 1969) and the values of the gravity-darkening coefficients $g_{1}=g_{2}=0.32$ (Lucy 1967) are used. The bolometric and passband-specific limb-darkening coefficients are chosen from Van Hamme (1993)'s table. 


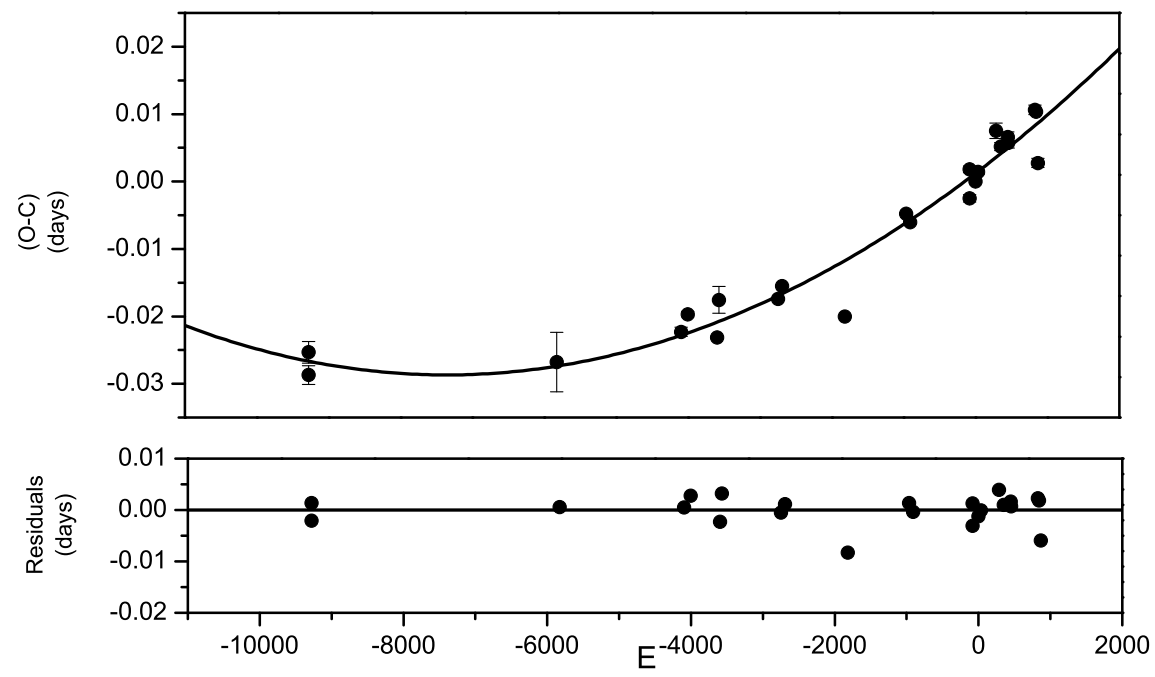

Fig. 2.- The $(O-C)$ values of II UMa from the linear ephemeris of Equation (1) is presented in the upper panel. The solid line in the panel refers to upward parabolic variation, which reveals a continuous increase in the orbital period. After the upward parabolic change were removed, the residuals are plotted in the lower panel.

The adjustable parameters are: the orbital inclination $i$; the mean temperature of star 2 $\left(T_{2}\right)$; the monochromatic luminosity of star $1\left(L_{1 B}, L_{1 V}, L_{1 R}\right.$ and $\left.L_{1 I}\right)$; the dimensionless potential of star $1\left(\Omega_{1}=\Omega_{2}\right.$ in mode 3 for overcontact configuration) and the third light $l_{3}$. The final photometric solutions are listed in Table 4 and the theoretical light curves are displayed in Fig. 3. The contact configuration of II UMa is displayed in Fig. 4.

It has to be mentioned that the errors listed in Table 4 are internal errors resulting from the application of the WD code to the supplied data. The effective temperature of the primary star $\left(T_{1}\right)$ may have some uncertainties due to the very wide spectral lines coming from the binary and the presence of the lines coming from the third body. We use the color index of II UMa to estimate the real uncertainties of $T_{1}$. According to the color index $(\mathrm{B}-\mathrm{V}$ $=0.447)$ given by the Tycho-2 Catalogue (Høg et al. 2000), the spectral type of II UMa is F5. However, the 2MASS All Sky Catalogue gives the color index of J - H = 0.189 (Cutri et al. 2003 ), which corresponds to a spectral type of F3. And the color index of V - K = 1.089 also supports that the spectral type is F5 (Cox 2000). Thus, the effective temperature of the primary star $\left(T_{1}\right)$ may range from $6550 \mathrm{~K}$ to $6680 \mathrm{~K}$. Solution B of $T_{1}=6680 \mathrm{~K}$ is also list in Table 4. According to Oh et al. (2007)'s work, we also set $T_{1}=6412 \mathrm{~K}$ and give Solution 
Table 4: Photometric solutions of II UMa

\begin{tabular}{llll}
\hline \hline Parameters & Solution A & Solution B & Solution C \\
\hline$T_{1}(K)$ & 6550 (fixed) & 6680 (fixed) & 6412 (fixed) \\
$g_{1}$ & 0.32 (fixed) & 0.32 (fixed) & 0.32 (fixed) \\
$g_{2}$ & 0.32 (fixed) & 0.32 (fixed) & 0.32 (fixed) \\
$A_{1}$ & 0.50 (fixed) & 0.50 (fixed) & $0.50($ fixed $)$ \\
$A_{2}$ & 0.50 (fixed) & $0.50($ fixed $)$ & 0.50 (fixed) \\
$\mathrm{q}\left(M_{2} / M_{1}\right)$ & 0.172 (fixed) & 0.172 (fixed) & 0.172 (fixed) \\
$i\left(^{\circ}\right)$ & $77.8( \pm 0.3)$ & $77.8( \pm 0.3)$ & $77.7( \pm 0.3)$ \\
$\Omega_{\text {in }}$ & 2.1615 & 2.1615 & 2.1615 \\
$\Omega_{\text {out }}$ & 2.0511 & 2.0511 & 2.0511 \\
$\Omega_{1}=\Omega_{2}$ & $2.0659( \pm 0.0047)$ & $2.0685( \pm 0.0057)$ & $2.0656( \pm 0.0048)$ \\
$T_{2}(K)$ & $6554( \pm 7)$ & $6684( \pm 8)$ & $6418( \pm 7)$ \\
$\Delta T(K)$ & 4 & 4 & 6 \\
$T_{2} / T_{1}$ & $1.001( \pm 0.001)$ & $1.001( \pm 0.001)$ & $1.001( \pm 0.001)$ \\
$L_{1} /\left(L_{1}+L_{2}\right)(B)$ & $0.8084( \pm 0.0008)$ & $0.8086( \pm 0.0007)$ & $0.8082( \pm 0.0008)$ \\
$L_{1} /\left(L_{1}+L_{2}\right)(V)$ & $0.8091( \pm 0.0010)$ & $0.8092( \pm 0.0010)$ & $0.8089( \pm 0.0010)$ \\
$L_{1} /\left(L_{1}+L_{2}\right)\left(R_{c}\right)$ & $0.8094( \pm 0.0015)$ & $0.8095( \pm 0.0015)$ & $0.8092( \pm 0.0015)$ \\
$L_{1} /\left(L_{1}+L_{2}\right)\left(I_{c}\right)$ & $0.8097( \pm 0.0030)$ & $0.8097( \pm 0.0030)$ & $0.8095( \pm 0.0030)$ \\
$L_{3} /\left(L_{1}+L_{2}+L_{3}\right)(B)$ & $0.2992( \pm 0.0023)$ & $0.2954( \pm 0.0023)$ & $0.3013( \pm 0.0023)$ \\
$L_{3} /\left(L_{1}+L_{2}+L_{3}\right)(V)$ & $0.2754( \pm 0.0032)$ & $0.2728( \pm 0.0032)$ & $0.2762( \pm 0.0032)$ \\
$L_{3} /\left(L_{1}+L_{2}+L_{3}\right)\left(R_{c}\right)$ & $0.2674( \pm 0.0051)$ & $0.2648( \pm 0.0051)$ & $0.2684( \pm 0.0050)$ \\
$L_{3} /\left(L_{1}+L_{2}+L_{3}\right)\left(I_{c}\right)$ & $0.2682( \pm 0.0098)$ & $0.2658( \pm 0.0098)$ & $0.2693( \pm 0.0097)$ \\
$r_{1}($ pole $)$ & $0.5209( \pm 0.0004)$ & $0.5241( \pm 0.0008)$ & $0.5219( \pm 0.0001)$ \\
$r_{1}($ side $)$ & $0.5790( \pm 0.0006)$ & $0.5842( \pm 0.0014)$ & $0.5807( \pm 0.0002)$ \\
$r_{1}($ back $)$ & $0.6083( \pm 0.0008)$ & $0.6148( \pm 0.0017)$ & $0.6104( \pm 0.0002)$ \\
$r_{2}($ pole $)$ & $0.2499( \pm 0.0005)$ & $0.2512( \pm 0.0011)$ & $0.2522( \pm 0.0002)$ \\
$r_{2}($ side $)$ & $0.2644( \pm 0.0006)$ & $0.2660( \pm 0.0013)$ & $0.2672( \pm 0.0002)$ \\
$r_{2}($ back $)$ & $0.3424( \pm 0.0024)$ & $0.3490( \pm 0.0057)$ & $0.3546( \pm 0.0010)$ \\
$f$ & $86.6 \%( \pm 4.2 \%)$ & $84.2 \%( \pm 5.2 \%)$ & $86.9 \%( \pm 4.3 \%)$ \\
$\Sigma \omega(O-C)^{2}$ & 0.036625 & 0.036671 & 0.036514 \\
\hline & & & \\
\hline & & &
\end{tabular}

Notes. The errors listed in Table 4 are internal errors resulting from the application of the WD code to the supplied data.

$\mathrm{C}$ in Table 4. We can conclude that Solution A, Solution B and Solution C give out almost consistent results although $T_{1}$ ranges from $6412 \mathrm{~K}$ to $6680 \mathrm{~K}$. 


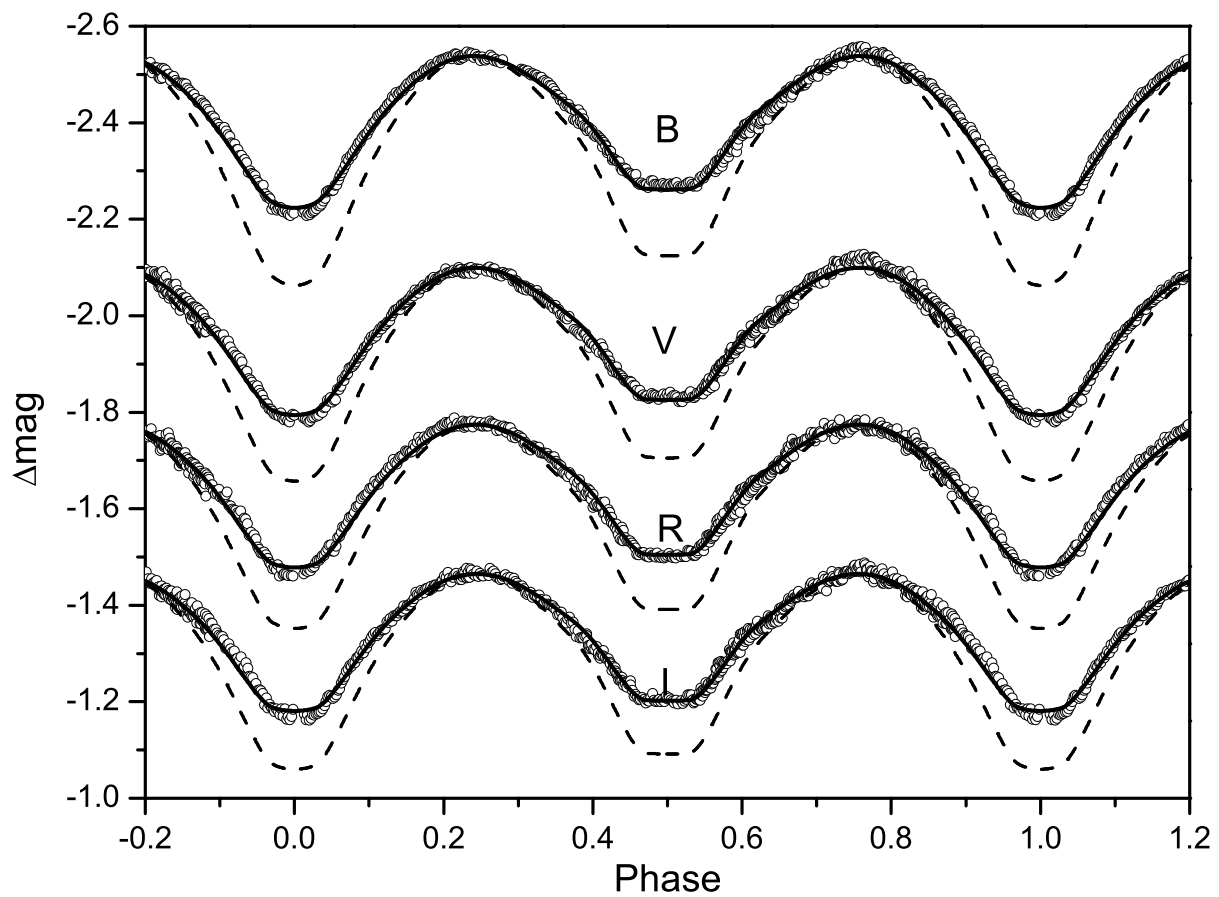

Fig. 3.- Observed (open circles) and theoretical (solid lines) light curves in the $B V R_{c}$ and $I_{c}$ bands for II UMa. The standard deviation of the fitting residuals are 0.011 mag for $B$ band, $0.011 \mathrm{mag}$ for $V$ band, $0.010 \mathrm{mag}$ for $R_{c}$ band and $0.010 \mathrm{mag}$ for $I_{c}$ band, respectively. The dash lines represent theoretical light curves without the third light.

\section{Discussions and Conclusions}

Light curves' solutions indicate that II UMa is an overcontact binary system with a quite high contact degree $(f=86.6 \%)$ and an extremely low mass ratio $(q=0.172)$, which indicate that it is at the final evolutionary stage of cool short-period binaries. It may merge into a single rapid-rotation star, which may be the progenitor of blue straggler or FK Comtype star (Zhou et al. 2015). The two components have nearly the same surface temperature $(\Delta T=4 K)$ in spite of their quite different masses and radii, which indicate that the system is under thermal contact. Considering the mass function given by Rucinski et al. (2002): $\left(M_{1}+M_{2}\right) \sin ^{3} i=2.180 \pm 0.080 M_{\odot}$ and the orbital inclination $\left(i=77.8^{\circ}\right)$ obtained by the light curves' solutions, the mass of the two components are calculated to be $M_{1}=$ $1.99( \pm 0.08) M_{\odot}, M_{2}=0.34( \pm 0.01) M_{\odot}$. Spectroscopic search carried out by D'Angelo et al. 


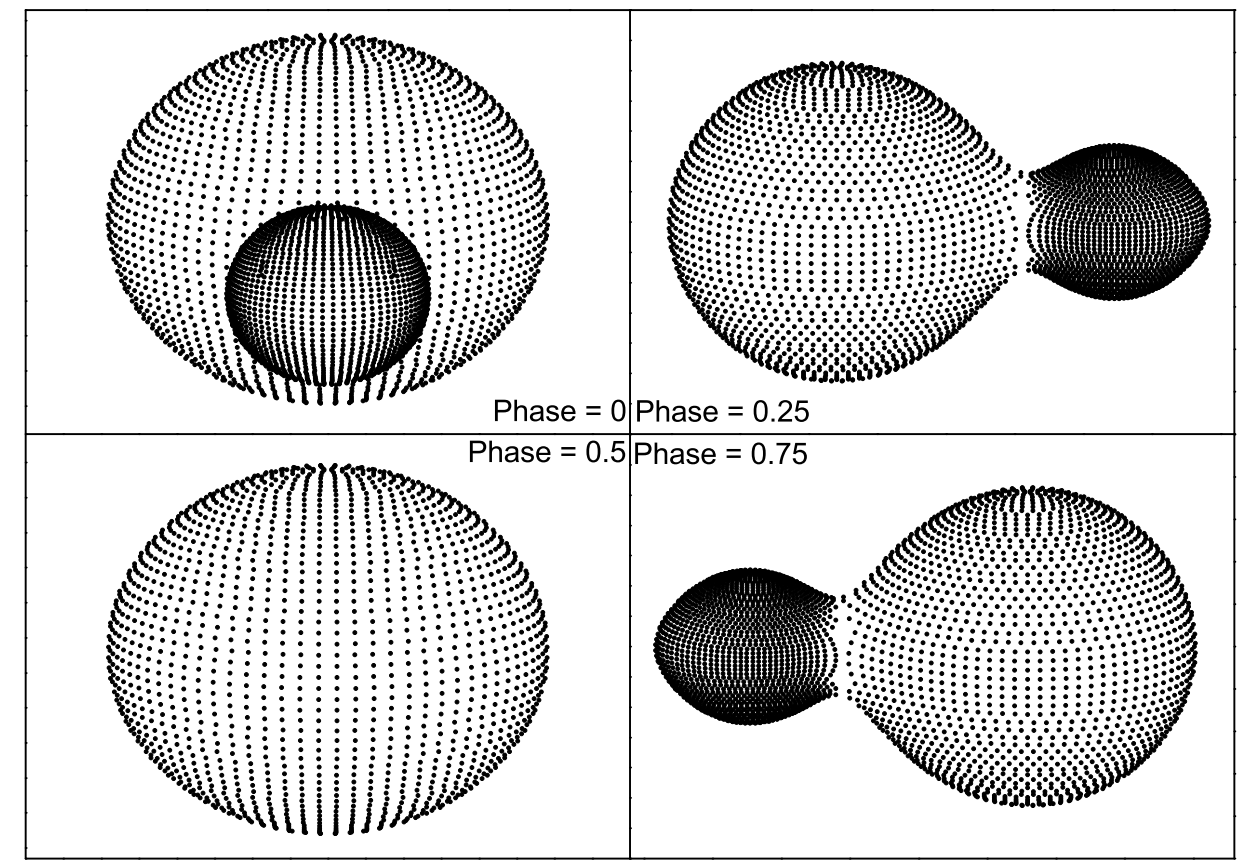

Fig. 4. - Contact configurations of II UMa at phase 0.0, 0.25, 0.5, 0.75 .

(2006) confirmed that II UMa was a triple system with a solar-type tertiary component, and determined the effective temperature of the third component to be $T_{3}=6100 \mathrm{~K}$, which corresponded to a G0V type star. The mass of the third component is estimated to be $M_{3}=1.34( \pm 0.05) M_{\odot}$ according to the mass ratio $\left(M_{3} / M_{1}=0.67\right)$ obtained. The third light $\left(l_{3}\right)$ is also included as an adjustable parameter during the photometric processing. The light curve solutions also confirm that it is a triple system and the third component contributes nearly a quarter of the total luminosity. As shown in Fig. 3, the existence of the third light reduces the occultation depth apparently.

Spectroscopic observations show that II UMa may be a contact binary with its primary component to be a giant star. It does have a quite long period $(\mathrm{P}=0.82522 \mathrm{~d})$ which does not obey the well-defined period-color relation of contact binary. However, the parameters obtained by us show that the radius of the apparent giant is equal to about $2.7 R_{\odot}$. The $2 M_{\odot}$ star should have a radius equal to about $3.5 R_{\odot}$ when it leaves MS. Thus, the primary star of II UMa has evolved from Zero Age Main Sequence (ZAMS), but it is still before Terminal Age Main Sequence (TAMS). II UMa is one of the A-type W UMa stars. Detailed modeling 
by Stepien (2011) concluded that initially detached binary systems will eventually evolve to main sequence contact binaries or Algol-type binary systems. We assume that II UMa is formed from an initially detached system via angular momentum loss (AML) by means of magnetic stellar wind. It is just under the late evolutionary stage of late-type tidal-locked binary stars, which might be close to merging and evolving into a single rapid-rotating star.

II UMa is a member of a visual binary system. The close binary system is even confirmed to be a triple system with a close-in solar-type tertiary component orbiting around the close binary system. Thus, it is actually a quadruple system. As discussed by Qian et al. (2013, 2014), the existence of an additional stellar component in the binary system may play an important role for the formation and evolution by removing angular momentum from the central binary system during the early dynamical interaction or late evolution. The $O-C$ curve analysis shows a continuous period increase at a rate of $d P / d t=4.88 \times 10^{-7} d a y \cdot y a r^{-1}$, which may be just a part of a cyclic period change, or the combinational period change of a parabolic variation and a cyclic one. More times of minimum light are needed to confirm this. II UMa is an important target for testing theories of star formation and stellar dynamical evolution and interaction. It is possible that third-body interactions in the birth environment may help to accelerate the orbital evolution of the central binary system. Angular momentum is drained from the inner close pair either by the ejection of the tertiary companion (Goodwin et al. 2004) or through the Kozai mechanism (Kozai 1962; Fabrycky \& Tremaine 2007).

We thank the anonymous referee for useful comments and suggestions that have improved the quality of the manuscript. This work is supported by the Chinese Natural Science Foundation (Grant No. 11133007 and 11325315), the Strategic Priority Research Program "The Emergence of Cosmological Structure" of the Chinese Academy of Sciences (Grant No. XDB09010202) and the Science Foundation of Yunnan Province (Grant No. 2012HC011). New CCD photometric observations of II UMa were obtained with the $60 \mathrm{~cm}$ and $1 \mathrm{~m}$ reflecting telescope at Yunnan Observatories (YNOs).

\section{REFERENCES}

Brat, L., Trnka, J., Smelcer, L., et al. 2011, Open European Journal on Variable Stars, 137, 1

Cox, A. N. 2000, Allen's Astrophysical Quantities (4th ed.; NewYork: Springer)

Cutri, R. M., Skrutskie, M. F., van Dyk, S., et al. 2003, VizieR Online Data Catalog, 2246, 
D'Angelo, C., van Kerkwijk, M. H., \& Rucinski, S. M. 2006, AJ, 132, 650

Drozdz, M., \& Ogloza, W. 2005, Information Bulletin on Variable Stars, 5623, 1

Dvorak, S. W. 2011, Information Bulletin on Variable Stars, 5974, 1

Eggen, O. J., \& Iben, I., Jr. 1989, AJ, 97, 431

Fabrycky, D., \& Tremaine, S. 2007, ApJ, 669, 1298

Goodwin, S. P., Whitworth, A. P., \& Ward-Thompson, D. 2004, A\&A, 414, 633

Høg, E., Fabricius, C., Makarov, V. V., et al. 2000, A\&A, 355, L27

K. Nagai et al. 2007, Variable Star Bulletin (Japan), 45, 1

K. Nagai et al. 2009, Variable Star Bulletin (Japan), 48, 1

Kozai, Y. 1962, AJ, 67, 591

Krajci, T. 2005, Information Bulletin on Variable Stars, 5592, 1

Lucy, L. B. 1967, ZAp, 65, 89

Nelson, R. H. 2005, Information Bulletin on Variable Stars, 5602, 1

Oh, K.-D., Kim, C.-H., Kim, H.-I., \& Lee, W.-B. 2007, The Seventh Pacific Rim Conference on Stellar Astrophysics, 362, 82

Porowski, C. H. 2005, Information Bulletin on Variable Stars, 5606, 1

Qian, S.-B., Liu, N.-P., Li, K., et al. 2013, ApJS, 209, 13

Qian, S.-B., Zhou, X., Zola, S., et al. 2014, AJ, 148, 79

Rucinski, S. M., Lu, W., Capobianco, C. C., et al. 2002, AJ, 124, 1738

Ruciński, S. M. 1969, Acta Astron., 19, 245

Stępień, K. 2006, Acta Astron., 56, 199

Stȩpień, K. 2011, Acta Astron., 61, 139

Van Hamme, W. 1993, AJ, 106, 2096

Van Hamme, W., \& Wilson, R. E. 2007, ApJ, 661, 1129 
Vilhu, O. 1982, A\&A, 109, 17

Wilson, R. E. 1979, ApJ, 234, 1054

Wilson, R. E. 1990, ApJ, 356, 613

Wilson, R. E., 2008, ApJ, 672, 575

Wilson, R. E., 2012, AJ, 144, 73

Wilson, R. E., Devinney E.J. 1971, ApJ, 166, 605

Wilson, R. E., Van, Hamme. W., Terrell, D., 2010, ApJ, 723, 1469

Yılmaz, M., Baştürk, Ö., Özavcı, İ., Şenavcı, H. V., \& Selam, S. O. 2015, New A, 34, 271

Zasche, P., Uhlar, R., Kucakova, H., Svoboda, P., \& Masek, M. 2014, Information Bulletin on Variable Stars, 6114, 1

Zhou, X., Qian, S.-B., Liao, W.-P., et al. 2015, AJ, 150, 83 